\title{
Statement of the Romanian Society of Pediatrics on the role of healthcare professionals in fighting smoking during childhood and adolescence
}

\author{
Doina Plesca', Oana-Cristina Marginean², Cristian Gheonea ${ }^{3}$, Sorin Man", \\ Ladislau Ritli ${ }^{5}$, Ioan Gherghina ${ }^{6}$, Aurel Nechita ${ }^{7}$, Sorin Buzinschi ${ }^{8}$, Liviu Pop ${ }^{9}$, \\ Otilia Marginean ${ }^{9}$, Claudia Olaru ${ }^{10}$, Smaranda Diaconescu ${ }^{10}$, Bogdan Stana ${ }^{10}$ \\ 1"Carol Davila" University of Medicine and Pharmacy, Bucharest, Romania \\ 2"University of Medicine, Science and Technology", Targu-Mures, Romania \\ ${ }^{3}$ University of Medicine and Pharmacy, Craiova, Romania \\ 4"Iuliu Hatieganu" University of Medicine and Pharmacy, Cluj-Napoca, Romania \\ ${ }^{5}$ University of Medicine, Oradea, Romania \\ ${ }^{6}$ National Institute for Mother and Child Health, \\ "Carol Davila" University of Medicine and Pharmacy, Bucharest, Romania \\ 7"Dunarea de Jos" University, Galati, Romania \\ ${ }^{8}$ University of Medicine, Sibiu, Romania \\ ${ }^{9}$ University of Medicine and Pharmacy, Timisoara, Romania \\ 10"Gr. T. Popa" University of Medicine and Pharmacy, lasi, Romania
}

Smoking is one of the major causes of death that can be prevented. In spite of the multiple approaches to fighting against smoking, the occurrence of smoking is hitting alarming rates throughout the world, among both adult and pediatric populations. The prevalence of smoking during childhood in Romania goes up to approximately $15 \%$, with teenagers forming the majority of smokers.

According to national statistics, the youngest reported age at the onset of smoking was 7 years old, and the mean age for such onset is 17.99 years old. The analysis of mean ages of onset for consumption of tobacco products indicate that more than half $(54.9 \%)$ of respondents stated they smoked for the first time between the ages of 15 and 19 years old. A worrying percentage was also obtained for the onset of such behavior before the age of 14 years old (16.4\%) (1).

The vast majority of smokers report that they started smoking during adolescence. As such, this age is the most susceptible to the smoking onset process, and from a health perspective pediatricians play a decisive role. The age of adolescence is the most vulnerable because of the continuous oscillations and the its characteristic indecisiveness.
Peer influence is essential for the developing personality of teenagers and can cause teenagers to very easily decide to start smoking.

The primary factor causing teenagers to start smoking is their peer group. It was thus proved that a teenager is more susceptible to start smoking if exposed to this factor. In addition to the peer group, there is also a genetic predisposition in terms of taking up smoking among people coming from families with nicotine receptor genotype (the genetic nature of the smoking addiction is a proven fact, with carriers of the mutation being unable to quit smoking).

From their point of view, healthcare professionals identify several types of smoking:

- Active ("first hand smoke") - which entails the direct use of products (classic cigarettes, electronic cigarettes, heated tobacco, other tobacco-based products)

- Passive ("second-hand smoke") - is the exposure of the child (fetus, new-born, infant, child) to cigarette smoke or directly to derivative products thereof

- "third-hand smoke" - the exposure of the child to tobacco derivative products (other- 
wise said, environmental pollution with noxious products after putting out a cigarette) (2)

Any healthcare professional working with teenagers has the obligation to and must have the capacity to identify at-risk youth (anamnesis) and provide minimal advice on the harmful effects of smoking. The approach to a smoking person, particularly in the case of teenagers, must employ agespecific questions; the information on smoking must be obtained while taking the patient's medical history for another medical reason, without hurting the teenager's pride, and in a manner that allows for plausible, real and honest answers.

A good time for educating or persuading teenagers about the harmful side effects of smoking is during their hospital admission (severe diseases, surgical interventions), when they are made aware of institutional restrictive measures and become quitting smoking.

In the case of smoking at pediatric ages, family members also play a major role. Family examples and support groups should provide parents with all the information on the medical effects smoking has on their children and should insist on the fact that the risk for these effects are inversely proportional to the age of smoking initiation. Such groups should organize sessions for parents that smoke, sessions for parents and children, as well as sessions only for children/teenagers that have already started to smoke (3). The Romanian Society of Pediatrics think that the family doctors role is essential both in the assessment of the caregivers (regarding the level of smoking and smoke-related diseases, chronic pathologies with familial aggregation as obesity, hyperlipidemia) and the children.

The media and particularly the Internet can be other useful methods for preventing or fighting against smoking at pediatric ages. Children and teenagers nowadays are increasingly more interested in the Internet, online gaming and computers in general. As such, it could be useful to create certain games that include smoking prevention strateconvinced that full recovery is only possible by

gies or characters that are designed so as to dissuade children and teenagers from starting to smoke.

It is necessary to create websites dedicated to this topic, which include guidelines for quitting smoking. Advertisements on the Internet could also be useful in preventing or fighting smoking. We think that media campaigns should be more focused on harmful potential side-effects of electronic devices, since they have an increasing popularity among teenagers. In Romania, at the present time, there is no media campaign focused on the potential harmful effects of e-cigarettes, and the vast majority of teens perceive this devices as being safe.

It is necessary to engage legislative bodies in order to find suitable and effective methods that have an impact on the prevention and fight against smoking at pediatric ages.

Another effective method in this respect could be introducing school classes for raising awareness among children on the negative effects of smoking. Such classes should be introduced as early as $5^{\text {th }}$ to $8^{\text {th }}$ grade and continued throughout high-school, comprising explanations adapted per age groups so as to get through to children and teenagers.

The Romanian Society of Pediatrics will increase its role in combating smoking in children and teenagers by organizing scientific sessions at symposiums / conferences / congresses / roundtables to address critical issues, by extending the national anti-smoking program (STOP FUMAT) in the field of pediatrics and by regular publishing in the Romanian Journal of Pediatrics of various materials against smoking.

The Romanian Society of Pediatrics will promote active measures in order to train healthcare professionals for a more effective fighting against smoking in children and teenagers. We strongly support the introduction of "Health Education" as mandatory in the scholar curricula, with a focus on smoking and other critical problems encountered in our country such as teen pregnancy.

Conflict of interest: none declared Financial support: none declared

\section{REFERENCES}

1. National Report on Drugs Situation in 2017, Ministry of Internal Affairs and National Antidrug Agency http://www.ana.gov.ro/rapoarte\%20nationale/sinteza_ RN_2017.pdf

2. Drehmer JE, Walters BH, Nabi-Burza E, Winickoff JP. Guidance for the Clinical Management of Thirdhand Smoke Exposure in the Child Health Care Setting. J Clin Outcomes Manag. 2017; 24(12):551-559.
3. Harvey J, Chadi N, Canadian Paediatric Society, Adolescent Health Committee. Preventing smoking in children and adolescents: Recommendations for practice and policy. Paediatr Child Health. 2016;21(4):209-21. 